\title{
Transcriptional regulation in microglia and neuropathic pain
}
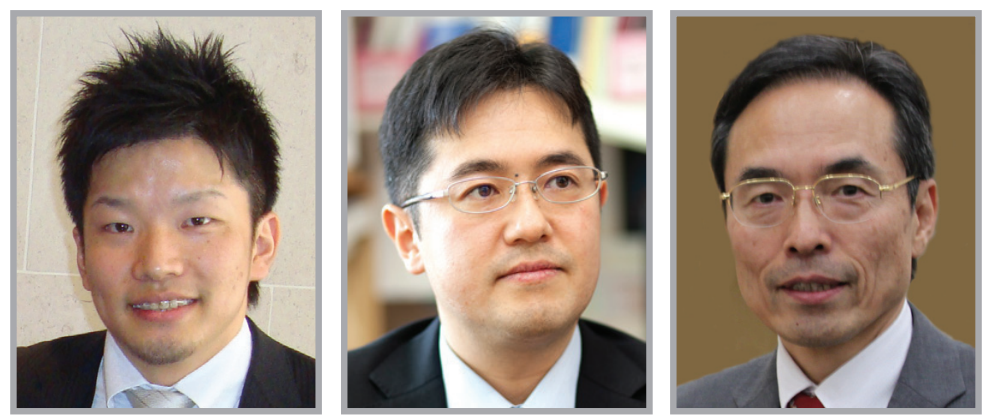

Takahiro Masuda ${ }^{1,2,3,4}$, Makoto Tsuda ${ }^{*, 1,2} \&$ Kazuhide Inoue $e^{* *, 1,4}$

First draft submitted: 5 June 2015; Accepted for publication: 3 July 2015; Published online: 25 February 2016

\section{Microglia \& neuropathic pain}

Microglia are the highly dynamic immune-related glial cells, also known as the specialized tissue macrophage of the CNS [1-3]. Under physiological conditions, these cells are characterized by small cell bodies with actively moving branched processes that survey the surrounding local environment for pathological alterations or disturbances to maintain brain homeostasis [4]. Because microglia have diverse roles during development, infection, aging and neurodegenerative disease [5], they thus exhibit a wide variety of phenotypes, thereby allowing specifically adapted functions, including phagocytosis and synapse remodeling $[1,6-7]$. Accumulating evidence has also demonstrated the crucial involvement of microglia in the pathogenesis of neuropathic pain $[8,9]$. In an animal model of neuropathic pain, peripheral nerve injury (PNI), the spinal dorsal horn where the injured nerve projects was found to contain reactive microglia, characterized by their state of morphological hypertrophy and proliferation $[8,9]$. Furthermore, these cells exhibit dramatic changes in the expression of various genes, including cell surface receptors for neurotransmission (e.g., nucleotide receptors) and innate responses (e.g., Toll-like receptors), and intracellular signaling molecules and bioactive diffusible factors (e.g., proinflammatory cytokines and neurotrophic factors) $[1,8]$. It is worth noting that pharmacological blockage or genetic manipulations of these molecules substantially decrease the excitability of the dorsal horn pain pathway, leading to suppression of pain hypersensitivity. Therefore, a phenotypic switch of microglia to a reactive state in the spinal dorsal horn is a crucial event

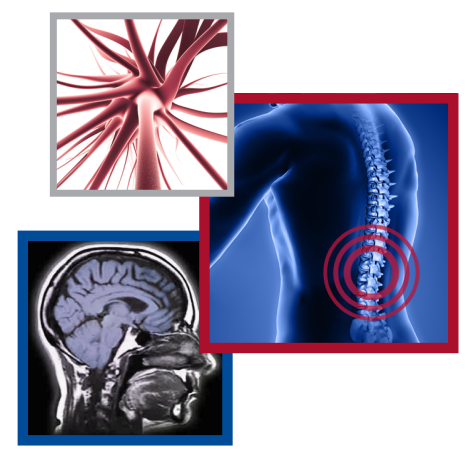

\section{KEYWORDS}

- ATP receptors • chronic pain

- glia • IRF • lentiviral vector

- proinflammatory cytokines $\bullet$ spinal cord $\bullet$ transcription factor

\section{"Microglia are the highly dynamic immune-related glial cells, also known as the specialized tissue macrophage of the CNS."}

'Department of Molecular \& System Pharmacology, Graduate School of Pharmaceutical Sciences, Kyushu University, 
“...microglia change their phenotypes according to the surrounding environment, resulting in either a positive or negative impact on physical conditions." that underlies the pathogenesis of neuropathic pain after PNI [8].

IRF8: a key transcription factor for microglial activation \& neuropathic pain Understanding the nature of reactive microglia is essential for the development of novel therapeutics for neuropathic pain. However, the molecular mechanisms by which microglia switch to reactive phenotypes remain elusive. The state of microglia is regulated by exogenous signals via surface receptors, and also tightly controlled by a subset of endogenous transcription factors. Recently, IRF8 was identified as a critical transcriptional regulator for microglial activation [10]. IRF8 is a member of the IRF family transcription factors (IRF1-9), and is expressed in immune cells such as lymphocytes and dendritic cells [11]. In the periphery, IRF8 has been shown to play pivotal roles in the immune system [11]. However, the roles of IRFs in the CNS are poorly understood. In the spinal cord, resting (surveying) microglia express low levels of IRF8, which is markedly upregulated following PNI [10]. Interestingly, IRF8 exhibits a restricted expression pattern in the spinal cord, where only microglial cells (and not neurons or astrocytes) express IRF8 [10]. The upregulation of microglial IRF8 after PNI has been shown to commence from postoperative day 1, peaking at day 3 until at least 3 weeks after the induced injury [10]. An in vitro gainof-function study utilizing primary cultured microglia with lentiviral-mediated forced expression of IRF8 revealed the interesting function of IRF8 in microglia; that is, ectopic expression of IRF8 promoted the transcription of genes associated with reactive states of microglia [10]. The genes affected are involved in microglial innate responses (TLR2), chemotaxis (the metabotropic purinoceptor $\mathrm{P}_{2} \mathrm{Y}_{12} \mathrm{R}$ and the chemokine receptor CX3CR1) and inflammatory components (IL-1 $\beta$, cathepsin S, BDNF and the ionotropic purinoceptor P2X4R) [10]. Importantly, a mutant IRF8 lacking the DNA-binding domain failed to produce the effects observed with wild-type IRF8 transduction [10], suggesting that IRF8 works in a manner that depends on its ability to bind DNA. The impact of IRF8 on microglial gene expression was further investigated by assessing loss-of-function phenotypes in IRF8-deficient mice [10]. PNI induced the upregulation of the above microglial genes in the spinal cord of wildtype mice, which was strongly inhibited in IRF8deficient mice [10]. However, this deficiency does not result in a global deregulation of the reactive processes of microglia because PNI-induced proliferation of spinal microglia was shown to not be affected by the absence of IRF8 [10]. Overall, IRF8 may activate the expression of a series of genes in spinal microglia after PNI, thereby inducing reactive phenotypes. Although whether IRF8 directly binds to promoter regions of these genes and induces their expression remains to be determined, these results provide a new mechanism for microglial activation.

Microglial IRF8 is required for neuropathic pain? IRF8-deficient mice were shown to have comparable basal mechanical sensitivity to wildtype mice but were resistant to PNI-induced tactile allodynia, abnormal pain hypersensitivity evoked by innocuous stimuli [10]. Furthermore, suppressing upregulated expression of spinal IRF8 (via the administration of small interfering RNA on day 5 and 6 post-PNI) in wild-type mice that had developed allodynia, resulted in a recovery from tactile allodynia [10]. These results indicate that the full development and maintenance of neuropathic pain require ongoing activity of IRF8 in spinal microglia after PNI. In contrast, IRF8-deficient mice showed comparable complete Freund's adjuvant or formalin-induced inflammatory pain to wild-type mice [10], suggesting that IRF8 is not involved in producing chronic inflammatory pain. Furthermore, transferring IRF8-overexpressing microglia spinally to normal mice has been shown to induce pain hypersensitivity [10], thus further demonstrating the impact of microglial IRF8 in modulating pain. However, this behavioral alteration was not observed in mice injected spinally with mutant microglial IRF8 [10]. Furthermore, pretreating IRF8-transduced microglia with a cocktail containing a function-blocking antibody for IL-1 $\beta$ and an inhibitor of cathepsin S attenuated IRF8 microglial-induced pain behavior [10]. Therefore, these results imply that inflammatory mediators derived from microglia that overexpress IRF8 produce allodynia. Overall, microglial IRF8 critically contributes to chronic neuropathic pain, but not chronic inflammatory pain, with minimal effect on basal sensory thresholds. These results may thus provide a new therapeutic strategy for neuropathic pain.

What mechanisms underlie the induction of IRF8 expression in microglia following PNI? Although IFN- $\gamma$ has been shown to increase the expression of IRF8 in vitro [12], mice lacking its receptor fail to prevent PNI-induced 
expression of IRF8 in spinal microglia [10]. Thus, an IFN- $\gamma$-independent mechanism may be involved. Indeed, upregulation of microglial IRF8 is observed in several animal models of neuronal injury, including hypoglossal nerve axotomy and kainic acid-induced neuronal injury [10]. In contrast, intraplantar complete Freund's adjuvant injection did not alter the expression of spinal cord IRF8 [10]. Therefore, the upregulation of IRF8 may be induced by molecules that are associated with nerve injury, but not with local tissue inflammation, which might be due to the different degrees of nerve damage in the sensory primary afferent between two models [13]. Considering IRF8 as a potential therapeutic target of neuropathic pain, a key step for the future will be determining the mechanisms of de novo expression of IRF8 in spinal microglia after PNI.

\section{Other transcription factors involved in determining microglial phenotype}

More recently, the other IRF family member IRF5 has been identified as a microglial transcription factor regulating a P2X4R-expressing phenotype [14]. In addition, PU.1, the ETS family transcription factor, is expressed in microglia of adult CNS tissue [15], and also controls function and phenotype of human brain microglia [16]. Furthermore, after PNI PU.1 expression is markedly increased in the spinal cord [17], where its expression is restricted in microglia [12]. Likewise, expression of Runx-1 has been reported to be increased in spinal microglia following PNI [18]. Therefore, these microglial transcription factors may concertedly control microglial phenotype.
However, the nature of these factors remain unknown and thus require further investigations, which may provide novel insights into the molecular mechanisms underlying microglial activation and neuropathic pain.

In conclusion, microglia change their phenotypes according to the surrounding environment, resulting in either a positive or negative impact on physical conditions. Therefore, targeting microglia-specific transcription factors involved in neuropathic pain as a therapeutic target may be more favorable for treating this condition.

\section{Key learning points}

PNI in mice or rats causes microglial activation in the spinal cord and long-lasting pain hypersensitivity in the ipsilateral hind paw.

\section{Financial \& competing interests disclosure}

This work was supported by grants from the Japan Society for the Promotion of Science (JSPS) through the 'Funding Program for Next Generation World-Leading Researchers (NEXT Program)' initiated by the Council for Science and Technology Policy (CSTP; M Tsuda) and the Ministry of Education, Culture, Sports, Science and Technology of Japan (T Masuda, M Tsuda, KInoue) and from the Japan Science and Technology Agency (JST) through the Core Research for Evolutional Science and Technology (CREST) program (K Inoue). The authors have no other relevant affiliations or financial involvement with any organization or entity with a financial interest in or financial conflict with the subject matter or materials discussed in the manuscript apart from those disclosed.

No writing assistance was utilized in the production of this manuscript.

\section{References}

1 Perry VH, Nicoll JA, Holmes C. Microglia in neurodegenerative disease. Nat. Rev. Neurol. 6(4), 193-201 (2010).

2 Ransohoff RM, Cardona AE. The myeloid cells of the central nervous system parenchyma. Nature 468(7321), 253-262 (2010).

3 Salter MW1, Beggs S. Sublime microglia: expanding roles for the guardians of the CNS. Cell 158(1), 15-24 (2014).

4 Hanisch UK, Kettenmann H. Microglia: active sensor and versatile effector cells in the normal and pathologic brain. Nat. Neurosci. 10(11), 1387-1394 (2007).

5 Prinz M, Priller J. Microglia and brain macrophages in the molecular age: from origin to neuropsychiatric disease. Nat. Rev. Neurosci. 15(5), 300-312 (2014).

6 Graeber MB. Changing face of microglia. Science 330(6005), 783-788 (2010).

7 Glass CK, Saijo K, Winner B, Marchetto MC, Gage FH. Mechanisms underlying inflammation in neurodegeneration. Cell 140(6), 918-934 (2010).

8 Inoue K, Tsuda M. Microglia and neuropathic pain. Glia 57(14), 1469-1479 (2009).

9 Calvo M, Bennett DL. The mechanisms of microgliosis and pain following peripheral nerve injury. Exp. Neurol. 234(2), 271-282 (2012).

10 Masuda T, Tsuda M, Yoshinaga R. IRF8 is a critical transcription factor for transforming microglia into a reactive phenotype. Cell Rep. 1(4), 334-340 (2012).

11 Tamura T, Yanai H, Savitsky D, Taniguchi T. The IRF family transcription factors in immunity and oncogenesis. Annu. Rev. Immunol. 26, 535-584 (2008).

12 Politis AD, Ozato K, Coligan JE, Vogel SN. Regulation of IFN-gamma-induced nuclear expression of IFN consensus sequence binding protein in murine peritoneal macrophages. J. Immunol. 152(5), 2270-2278 (1994).

13 Allchorne AJ, Gooding HL, Mitchell R, Fleetwood-Walker SM. A novel model of combined neuropathic and inflammatory pain displaying long-lasting allodynia and spontaneous pain-like behaviour. Neurosci. Res. 74(3-4), 230-238 (2012). 
EDITORIAL Masuda, Tsuda \& Inoue

14 Masuda T, Iwamoto S, Yoshinaga R et al. Transcription factor IRF5 drives P2X4R+reactive microglia gating neuropathic pain. Nat. Commun. 5, 3771 (2014).

15 Horiuchi M, Wakayama K, Itoh A et al. Interferon regulatory factor $8 /$ interferon consensus sequence binding protein is a critical transcription factor for the physiological phenotype of microglia. J. Neuroinflammation 9, 227 (2012).

16 Smith AM, Gibbons HM, Oldfield RL et al. The transcription factor PU. 1 is critical for viability and function of human brain microglia. Glia 61(6), 929-942 (2013).

17 Imai S, Ikegami D, Yamashita A et al. Epigenetic transcriptional activation of monocyte chemotactic protein 3 contributes to long-lasting neuropathic pain. Brain 136(Pt 3), 828-843 (2013).

18 Zusso M, Methot L, Lo R, Greenhalgh AD, David S, Stifani S. Regulation of postnatal forebrain amoeboid microglial cell proliferation and development by the transcription factor Runx1. J. Neurosci. 32(33), 11285-11298 (2012). 